\title{
MicroRNA in modern genetics
}

\author{
Thapa JB ${ }^{1}$ \\ ${ }^{I}$ Consultant Pathologist, Himal Hospital Pvt. Ltd., Kathmandu, Nepal
}

\section{Keywords: \\ miRNA; \\ mRNA; \\ oncogenesis}

\begin{abstract}
In recent years microRNAs have emerged as important players in modern genetics. This review attempts to introduce the biogenesis of microRNA and its important physiological role in protein synthesis. The association of microRNA with different cancers is discussed. Lastly the frontier field of therapy based on microRNAs to treat different diseases is introduced.
\end{abstract}

\section{INTRODUCTION}

Cutting edge advances are being made in the field of oncogenesis as better understanding of the cellular physiology of protein synthesis has been made in the last decade. There is no doubt that the cellular function of DNA is important. However its role is limited to long term stable storage of genomic material. The roles of different RNAs are emerging and they are varied and intricate. Dysfunction of miRNA can lead to many inborn and acquired diseases including many cancers. It is expected that the future will see even more discoveries in basic cellular physiology. This all can mean major changes will be seen in the diagnosis and management of many diseases. Therefore the objective of this paper is to introduce the miRNAs to the busy reader who is likely to encounter them even more frequently in the future.

\section{Abbreviations:}

$\mathrm{AMO}=$ anti-miRNA oligonucleotide, DSCR8P $=$ DiGeorge syndrome critical region 8 protein, MiRNA, miRNA = microRNA, $\mathrm{mRNA}=$ messenger $\mathrm{RNA}, \mathrm{OG}=$ oncogene, $\mathrm{nt}=$ nucleotide, $\mathrm{RISC}=\mathrm{RNA}$ inducing silencing complex,

\footnotetext{
Correspondence:

Dr. Jung Bahadur Thapa

Consultant Pathologist,

Pathology Laboratory, Himal Hospital Pvt. Ltd., Kathmandu, Nepal.

Emailjungbahadurthapa@gmail.com
}

siRNA $=$ small interfering $\mathrm{RNA}, \mathrm{tRNA}=$ transfer $\mathrm{RNA}$, ssRNA $=$ single stranded RNA, TSG $=$ tumour suppressor gene, UTR = untranslated region,

Nomenclature: e.g. (small r) mir-123 = immature miRNA e.g. pre-miRNA, (capital R) miR-789 = mature miRNA, miR345* $=$ asterisk indicates low levels of miRNA expression.

\section{History of RNAs}

Life on earth has existed for about 4 billion years. The earliest life forms have been assumed to be simple peptide chains which later differentiated into more complex nucleotides possibly the early forms of RNAs. ${ }^{1}$

\section{The importance of RNA}

The RNAs are more primitive and also functionally more versatile than the better known DNA. Like DNA they are capable of storing genetic information in shorter temporary bits. However unlike DNA they also have unique catalytic enzymatic functions. This gave RNA the special primordial capability of self replication. Some life forms still exists with RNA only, like the present day RNA viruses. However the initial primitive life forms that existed with only RNA and without DNA were relatively unstable. The hydroxyl groups of RNA were frequently hydrolyzed by external xenotoxic agents. With evolutionary adaptations the hydroxyl group 
was gradually deleted, thymine was substituted for uracil, and some of the single stranded RNAs were converted to the more stable and longer double stranded DNA. ${ }^{2}$

It has long been known that DNA holds the secure core repository of genetic information and RNA makes copies of the information as required for protein synthesis. However it is now known that DNA is subject to influences both by histone originated epigenetic control and indirectly through microRNA (miRNA) induced messengerRNA (mRNA) suppression.

\section{Landmarks in the study of the RNAs}

Historically in 1868 Frederich Meischer discovered nucleic acids in the cell nuclei, which he called "nuclein" a term now, abandoned. ${ }^{3}$ Throughout the last half of the 20th century and the first decade of the 21 st century spectacular major breakthroughs were achieved in RNA research, and they were rightfully recognized by society with the award of five prestigious Nobel Prizes.

In 1959 Severo Ochoa and Arthur Kornberg won the Nobel Prize for discovering an enzyme that synthesizes RNA in the laboratory. Robert Holley discovered the 77 nucleotides of the yeast transfer RNA ( $t$-RNA) and won the Nobel Prize in 1968. Retroviruses and reverse transcriptase were discovered by David Baltimore, Renalto Delbecco, and Howard Temin and they received the Nobel Prize in 1975. Introns and RNA slicing was discovered by Philips Sharp and Richard Roberts and they were also awarded the Nobel Prize in 1993.

MiRNA were discovered in 1993 by Victor Ambros, Rosalind Lee and Rhonda Feinbaum in the study of the gene lin-14 in Caenorhabditis elegans. ${ }^{4}$ Studies in RNA induced interference resulted in the 2006 Nobel Prize for Andrew Fire and Craig Mello. The basic types of RNAs are shown in fig.1.

\section{MicroRNAs in health}

MicroRNAs are short chained single stranded RNA (ssRNA) 19-25 nt (average length $22 \mathrm{nt}$ ) derived from hairpin shaped transcripts seen in eukaryotic cells. They are post-transcriptional negative cellular regulators that bind to mRNA. They normally participate in cellular development, differentiation, proliferation, and apoptosis and hence are of prime importance for optimal cellular function. They regulate gene function by fine tuning mRNA function turning off protein synthesis as per body requirements. They cause translation repression, target degradation or gene silencing. They have an intricate system of functioning. MiRNA have multiple binding sites and each site can have multiple binding receptors. The human genome may code for over 1000 miRNA. ${ }^{5}$ They can target up to $60 \%$ of human genes. ${ }^{6}$ They are normally present in many varied human

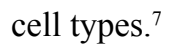

\section{Biogenesis of microRNAs (fig.2)}

In the cell nuclei miRNA genes present in the genome are transcribed by RNA polymerase II and sometimes RNA polymerase III into long primary miRNA (pri-miRNA), ${ }^{8,9}$ Drosha an intra-nuclear RNA polymerase III converts primary miRNA into pre-miRNA. ${ }^{10}$ Drosha acts with DiGeorge syndrome critical region 8 protein (DSCR8P) for this purpose. ${ }^{11}$ DSCR8P is also known as Pasha in invertebrates. An alternate pathway involving introns producing pre-miRNA called mirtron pathway through the spliceosome is also present. ${ }^{12}$

Pre-miRNA is sent from the nucleus into the cytoplasm by the help of exportin5/RanGTP hetero-complex. ${ }^{13}$ In the cytoplasm another RNA polymerase III called Dicer converts Pre-miRNA into 21-22 nt long siRNA (miRNA/ miRNA) duplexes. Only one strand of this duplex is used for the formation of RNA inducing silencing complex (RISC). These single miRNA strands are collected as Argonaute proteins (Ago1-4), and eventually form mature active RISC. Argonaute proteins are the catalytic part of RISC. The RISC/Ago 1-4 complex is responsible for mRNA silencing (fig. 3).

\section{Mechanism of RNA inducing silencing complex (RISC)}

RISC targets 3' untranslated region (UTR) rather than the 5' UTR of mRNA to suppress protein synthesis.14 Six models of translational repression of mRNA by miRNA have been proposed (fig. 4). They include:

a. RISC induces de-adenylation which causes decrease of translational efficiency by blocking the target mRNA circularization $^{15}$

b. RISC blocking cap function by suppressing cap eIF4E ${ }^{16}$

c. RISC blocking a late step of translation like recruitment of $60 \mathrm{~S}$ ribosomal subunit ${ }^{17}$

d. RISC blocking a post-initiation step such as elongation or ribosome drop-off ${ }^{18}$

e. RISC causes proteolysis of new peptides during the translation ${ }^{19}$

f. RISC collects target mRNAs to processing bodies, in which mRNA is degraded and/or stored as inactive forms $^{20}$

\section{Role of microRNA in cancer}

MiRNA dysfunction is now known to be associated with different types of cancers in man. A database of diseases associated with miRNA diseases is available to the public. ${ }^{21}$ MiRNA signatures can be used to diagnose, prognosticate and are being currently investigated to help treat malignancies. In the last decade a new set of cancer regulators have been identified. They are oncomirs and anti-oncomirs. Oncomirs 
negatively regulate tumour suppression genes (TSG), while anti-oncomirs negatively suppress oncogenes (OG). It is well established that abnormalities of TSG and OG are important recognized events in oncogenesis. Defective MiRNAs forms can act as oncomirs or anti-oncomirs and thus contributing to the oncogenesis.

There are a few mechanisms proposed for aberrant miRNA synthesis. Chromosomal translocations or point mutations in the genome can lead to abnormal primary miRNA formation and resultant abnormal miRNA synthesis. Mutations in miRNA can lead to abnormal RISC functions. Mutations or single nucleotide polymorphism, (SNP) in miRNA or in the seed region of the target mRNA can hinder proper target recognition. Therefore genes can escape from the normal suppressive regulatory action of RISC. All these factors can contribute to oncogenesis.

It is also known that the cell regulator p53, also known as the guardian angel of the cell, normally protects the cell by activating miR34, a miRNA. ${ }^{22}$ Dysfunction of miR34 can thus lead to defects in defensive apoptotic mechanism, and DNA damaged cells that should have been removed by apoptosis persist in the body leading to tumours.

Abnormalities of miRNA have been detected with different neoplasm. Let-7 is an anti-oncomir that normally regulates post-transcriptional RAS oncogene involved in lung cancer. 23 Lymphoma karyotyping reveals miR-19 an oncomir. ${ }^{24}$ Defects in an anti-oncomir cluster miR-15a/miR16-A have been identified in chronic lymphatic leukaemia. ${ }^{25}$ In breast, colon, lung and pancreatic cancers an oncomir miR-155 is over expressed. ${ }^{26}$ More examples are listed in Table 1.

\section{Laboratory testing of miRNA}

MiRNA can be tested by semi-quantitatively or quantitatively by a two step test process. The first step consists of a modified RT-PCR followed by a second step real time quantitative PCR. ${ }^{27}$ Levels of miRNA can also be determined by microarray techniques hybridizing hundreds or thousands of miRNA probes on slides or chips. ${ }^{28}$ Northern blot analysis which is a well-established technique for studying messenger RNA expression and was soon adapted to detect miRNAs in cells or tissues. ${ }^{29}$

\section{MiRNA related therapeutics}

Many strategies are being evaluated to reverse the changes induced by miRNA dysfunction. Anti-miRNA oligonucleotides (AMO, antagomirs) have been developed for miRNA inhibition. Three types of AMOs include: 2'OMe, 2"MOE, and locked nucleic acid (LNA). Another strategy is suppression of the miRNA biogenic pathway by inhibiting Drosha or Dicer RNA polymerases which reduces mature miRNA levels. ${ }^{30}$ Lastly replacement of defective miRNA by normal miRNA in diseased tissue has

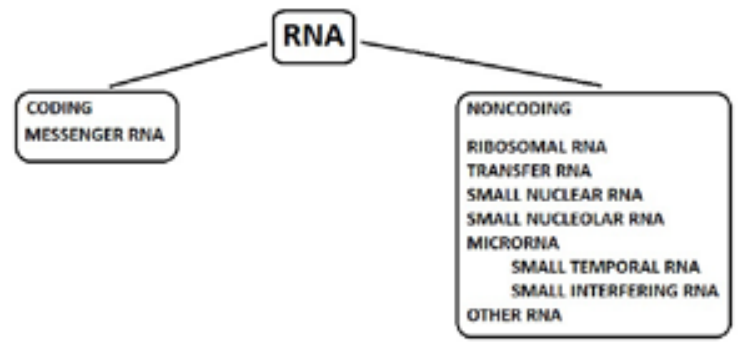

Figure 1: Basic types of RNA

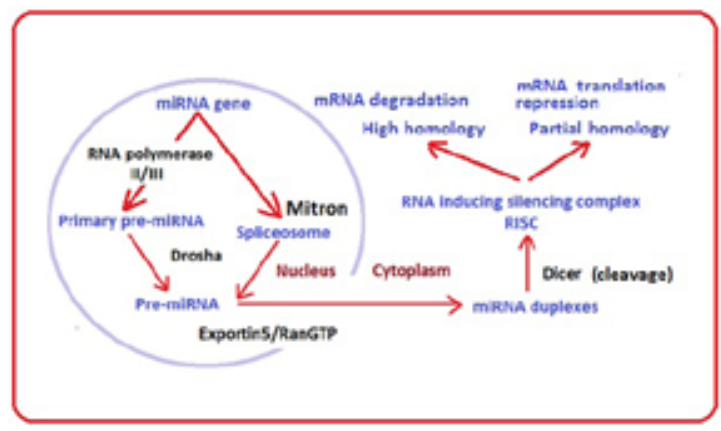

Figure 2: Simplified diagram of the biogenesis of miRNA and RISC

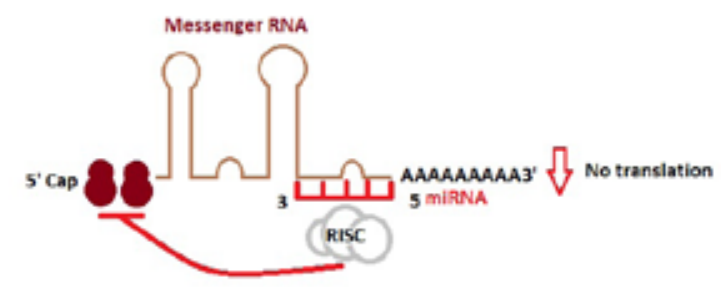

Figure 3: miRNA silencing of mRNA at 3'end.

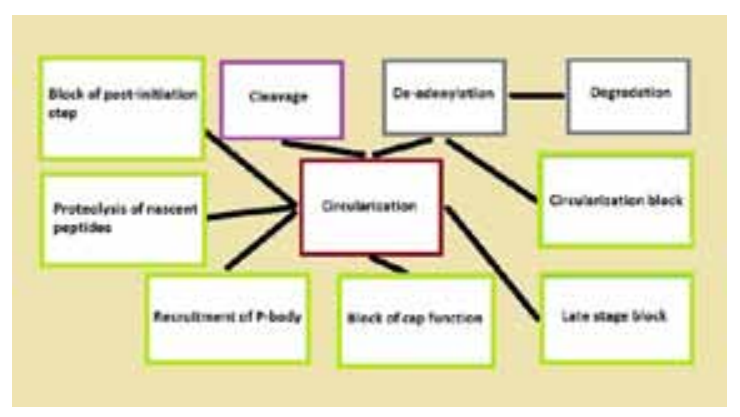

Figure 3: Proposed mechanisms of translational regression by $\mathrm{miRNA}$ 
also been investigated into. ${ }^{31}$ However at present miRNA therapy is still in its early stages and will take some time before gaining full clinical acceptance.

\section{CONCLUSION}

Understanding the role of miRNA in health and disease is still in its early days. It is known that disruption of any stage of biogenesis of miRNA can be associated with disease or neoplasm. Efforts are being made to develop treatment strategies tailored on these specific defects. We can anticipate more developments in the field in the future.

\section{REFERENCES}

1. Orgel L. "Prebiotic chemistry and the origin of the RNA world". Crit Rev Biochem Mol Biol 2004;39:99-123.

2. Berg JM, Tymoczko JL, Stryer L editor. Biochemistry. 5th ed. WH Freeman and Company.202. pp78.

3. Dahm R (2005).Friedrich Miescher and the discovery of DNA. Developmental Biology 2005; 15:274-88.

4. Lee RC, Feinbaum RL, Ambros V. The C. elegans heterochronic gene lin-4 encodes small RNAs with antisense complementarity to lin-14. Cell 1993;75:843-54.

5. Bentwich I, Avniel A, Karov Y et al. Identification of hundreds of conserved and nonconserved human microRNAs. Nat. Genet. 2005;37:766-70.

6. Friedman RC, Farh KK, Burge CB, Bartel DP. Most mammalian mRNA are conserved targets of microRNAs. Genome Res. 2009;19:92-105.

7. Lim LP, Lau NC, Weinstein EG et al. The microRNAs of Caenorhabditis elegans. Genes Dev.2003;17:991-1008.

8. Lee Y, Kim M, Han J et al. MicroRNA genes are transcribed by RNA polymerase II. EMBO J 2004;23:4051-60.

9. Borchert GM, Lanier W, Davidson BL. RNA polymerase III transcribes human microRNAs. Nat Struct Mol Biol 2006;13:1097101.

10. Lee Y, Ahn C, Han J et al. The nuclear RNase III Drosha initiates microRNA processing. Nature 2003;425:415-9.

11. Han J, Lee Y, Yeom KH, Kim YK, Jin H, Kim VN. The DroshaDGCR8 complex in primary microRNA processing. Genes Dev 2004;18:3016-27.

12. Ruby JG, Jan $\mathrm{CH}$, Bartel DP. Intronic microRNA precursors that bypass Drosha processing. Nature 2007;448:83-6.

13. Bohnsack MT, Czaplinski K, Gorlich D. Exportin 5 is a RanGTPdependent dsRNA-binding protein that mediates nuclear export of pre-miRNAs. RNA 2004; 10:185-91.

14. Gu S, Jin L, Zhang F, Sarnow P, Kay MA. Biological basis for restriction of microRNA targets to the 3' untranslated region in mammalian mRNAs. Nat Struct Mol Biol 2009;16:144-50.

15. Beilharz TH, Humphreys DT, Clancy JL et al. microRNA-mediated messenger RNA deadenylation contributes to translational repression in mammalian cells. PLoS one 2009;4:6783.

16. Mathonnet G, Fabian MR, Svitkin YV et al. MicroRNA inhibition of translation initiation in vitro by targeting the cap-binding complex eIF4F. Science 2007;317:1764-7.

17. Wang B, Yanez A, Novina CD. MicroRNA-repressed mRNAs contain 40S but not 60S components. Proc Natl Acad Sci 2008;105:5343-8.

18. Seggerson K, Tang L, Moss EG. Two genetic circuits repress the Caenorhabditis elegans heterochronic gene lin-28 after translation initiation. Dev Biol 2002;243:215-25.

19. Nottrott S, Simard MJ, Richter JD. Human let-7a miRNA blocks protein production on actively translating polyribosomes. Nat Struct Mol Biol 2006;13:1108-14.

20. Liu J, Valencia-Sanchez MA, Hannon GJ, Parker R. MicroRNAdependent localization of targeted mRNAs to mammalian P-bodies. Nat Cell Biol 2005;7:719-23.

21. Jiang Q, Wang Y, Hao Y et al. miR2Disease: a manually curated database for microRNA deregulation in human disease. Nucleic Acids Research 2009;37:D98-104.

22. He L, He X, Lim LP, de Stanchina E et al. A microRNA component of the p53 tumour suppressor network. Nature 2007;447:1130-4.

23. Johnson SM, Grosshans H, Shingara J et al. RAS is regulated by the let-7 microRNA family. Cell 2005;120:635-47.

24. Mavrakis KJ, Wolfe AL, Oricchio E et al. Genome-wide RNAmediated interference screen identifies miR-19 targets in Notchinduced T-cell acute lymphoblastic leukaemia. Nat Cell Biol 2010;12:372-9.

25. Calin GA, Ferracin M, Cimmino A et al. A microRNA signature associated with prognosis and progression in chronic lymphocytic leukemia. N Engl J Med 2005;353:1793-801.

26. Faraoni I, Antonetti FR, Cardone J, Bonmassar E. miR-155 gene: a typical multifunctional microRNA. Biochim Biophys Acta 2009; 1792:497-505

27. Caifu, Chen; Dana A. Ridzon et al. Real-time quantification of microRNA by stem-loop RT-PCR. Nucleic Acids Research 2005;33:E179.

28. Shingara J, Keiger K, Shelton J et al. An optimized isolation and labeling platform for accurate microRNA expression profiling. RNA 2005;11:1461-70.

29. Valoczi A, Hornyik C, Varga N, Burgyan J, Kauppinen S, Havelda Z. Sensitive and specific detection of microRNAs by Northern blot analysis using LNA-modified oligonucleotide probes. Nucleic Acids Res 2004;32:e175.

30. Soifer HS, Rossi JJ, Saetrom P. MicroRNAs in disease and potential therapeutic applications. Mol. Ther. 2007;15:2070-9.

31. Xiao J, Yang B, Lin H, Lu Y, Luo X, Wang Z. Novel approach for gene-specific interference via manipulating actions of microRNAs: examination on the pacemaker channel genes HCN2 and HCN4. J.Cell.Physiol 2007; 212:285-92. 